\title{
Inclusive Design for Brain Body Interfaces
}

\author{
Paul Gnanayutham ${ }^{1}$, Jennifer George ${ }^{2}$ \\ ${ }^{1}$ Department of Computing, University of Portsmouth, Buckingham Building, Lion Terrace, \\ Portsmouth, PO1 3HE, United Kingdom \\ ${ }^{2}$ SAE Institute, United House, North Road, London, N7 9DP, United Kingdom \\ \{paul.gnanayutham@port.ac.uk,jennifer.george@sae.edu\}
}

\begin{abstract}
In comparison to all types of injury, those to the brain are among the most likely to result in death or permanent disability. A certain percentage of these brain-injured people cannot communicate, recreate, or control their environment due to severe motor impairment. This group of individuals with severe head injury has received little from assistive technology. Brain computer interfaces have opened up a spectrum of assistive technologies, which are particularly appropriate for people with traumatic brain-injury, especially those who suffer from "locked-in" syndrome. Previous research in this area developed brain body interfaces so that this group of brain-injured people can communicate, recreate and launch applications communicate using computers despite the severity of their brain injury, except for visually impaired and comatose participants. This paper reports on an exploratory investigation carried out with visually impaired using facial muscles or electromyography (EMG) to communicate using brain body interfaces.
\end{abstract}

Keywords: Brain-Body Interface, Inclusive design, Neuro-rehabilitation, Assistive Technology and visual impairment, EEG, EMG and EOG.

\section{Introduction}

As medical technology not only extends our natural life span but also leads to increased survival from illness and accidents, the number of people with disabilities is constantly increasing. World Health Organization [1] estimates that there are more than 600 million people who are disabled as a consequence of mental, physical or sensory impairment thus creating one of the world's largest minorities. It has been estimated that 80 to 120 million European citizens have some form of disability, exceeding the population of almost every European state [2] In comparison to different types of injury, those to the brain are among the most likely to result in death or permanent disability. In the European Union, brain injury accounts for one million hospital admissions per year. A certain percentage of these brain-injured people cannot communicate, recreate, or control their environment due to severe motor 
impairment. This group of severely head injured people is cared for by nursing homes that cater for their wellbeing in every possible way. Their loved ones also play a major role in the wellbeing of this group of people.

\subsection{Brain Injury}

There are two stages in traumatic brain injury, the primary and the secondary. The secondary brain injury occurs as a response to the primary injury. In other words, primary brain injury is caused initially by trauma amyotrophic lateral sclerosis, brain stem stroke etc., but includes the complications, which can follow, such as damage caused by lack of oxygen, and rising pressure and swelling in the brain. A brain injury can be seen as a chain of events beginning with the first injury which occurs in seconds after the accident and being made worse by a second injury which happens in minutes and hours after this, depending on when skilled medical intervention occurs. There are three types of primary brain injury - closed, open and crush. Closed head injuries are the most common type, and are so called because no break of the skin or open wound is visible. Open head injuries are not so common. In this type of injury the skull is opened and the brain exposed and damaged. In crush injuries the head might be caught between two hard objects. This is the least common type of injury, and often damages the base of the skull and nerves of the brain stem rather than the brain itself. Individuals with brain injury require frequent assessments and diagnostic tests [3]. Most hospitals use the Glasgow Coma Scale for predicting early outcome from a head injury, for example, whether the person will survive or Rancho Levels of Cognitive Functioning for predicting later outcomes of head injuries [4].

\subsection{Brain Body Interface Devices}

The brain is the centre of the central nervous system in humans as well as the primary control centre for the peripheral nervous system (Fig.1.). The building blocks of the brain are special cells called neurons. The human brain has approximately hundred billion neurons. Neurons are the brain cells responsible for storing and transmitting information from a brain cell. The adult brain weighs three pounds and is suspended in cerebrospinal fluid. This fluid protects the brain from shock. The brain is also protected by a set of bones called the cranium or a skull. 


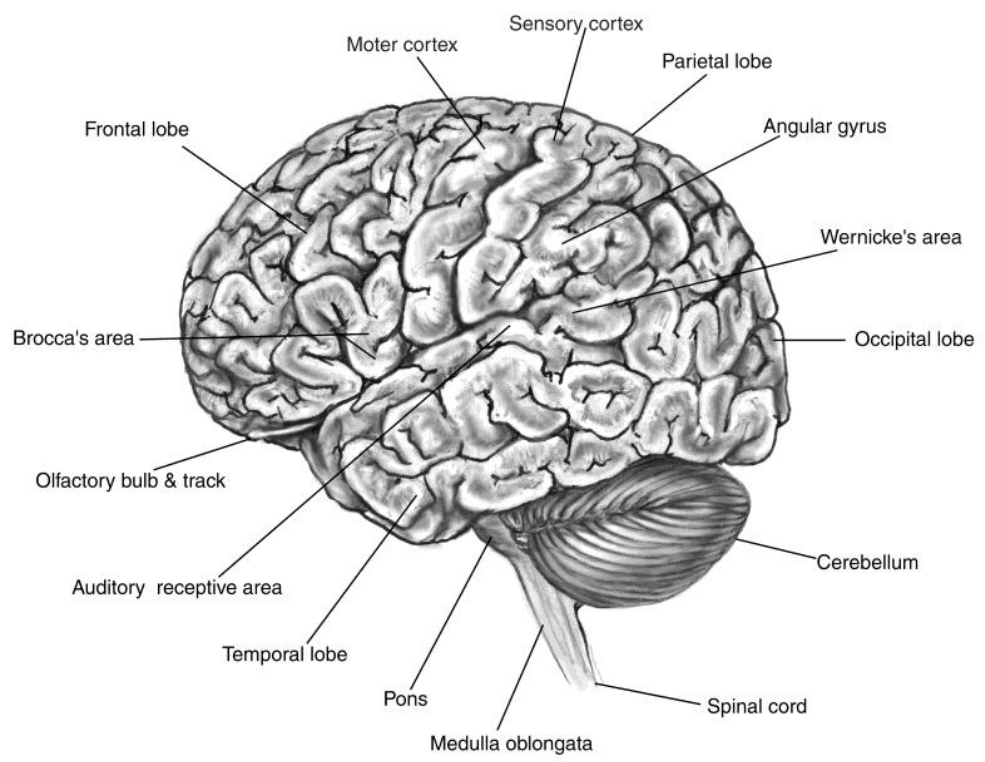

Fig. 1. Brain Map (Courtesy of www.headinjury.com)

The three main components of the brain are the cerebellum, cerebrum and brainstem. The cerebellum is located between the brainstem and the cerebrum. The cerebellum controls facial muscle co-ordination and damage to this area affects the ability to control facial muscles thus affecting signals (eye movements and muscle movements) needed by Brain-Body Interfaces. The cranial nerves that carry the signals to control facial movements also originate in the brainstem, hence the brainstem is of interest when using Brain-Body Interfaces.

Assistive devices are essential for enhancing quality of life for individuals with severe disabilities such as quadriplegia, amyotrophic lateral sclerosis (ALS), commonly referred to as Lou Gehrig's disease or brainstem strokes or traumatic brain injuries (TBIs). Research has been carried out on the brain's electrical activities since 1925 [5]. Brain-computer interfaces (BCIs), also called brain-body interfaces or brain-machine interfaces provide new augmentative communications channels for those with severe motor impairments. In 1995 there were no more than six active brain computer interface research groups, in 2000 there were more than twenty and now more than thirty laboratories are actively researching in BCI [6]. A BCI is a communication system that does not depend on the brain's normal output pathways such as speech or gestures but by using electrophysiological signals from the brain as defined by Wolpaw [7]. There are two types of brain body interfaces namely invasive (signals obtained by surgically inserting probes inside the brain) and non-invasive (electrodes placed externally on part of the body).

Brain activity produces electrical signals that can be read by electrodes placed on the skull, forehead or other part of the body (the skull and forehead are predominantly used because of the richness of bio-potentials in these areas). Algorithms then 
translate these bio-potentials into instructions to direct the computer, so people with brain injury have a channel to communicate without using the normal channels.

Non-invasive technology involves the collection of control signals for the brain computer interface without the use of any surgical techniques, with electrodes placed on their face, skull or other parts of their body. The non-invasive devices show that, signals obtained are first amplified, filtered and thereafter converted from analogue to digital signal. Various electrode positions are chosen by the developers, who choose electrode caps, electrode headbands with different positions and number of electrodes or the international 10-20 system [8]. Authorities dispute the number of electrodes needed for collection of usable bio-potentials [9]. There is only one agreed standard for the positions and number of electrodes that is the International 10-20 system of electrodes [10]

Invasive electrodes can give better noise to signal ratio and obtain signals from a single or small number of neurons. Signals collected from the brain require expensive and dangerous measures such as surgery. Neurons are the brain cells responsible for storing and transmitting information from a brain cell. Any mental experience even if unconscious has a signal associated with it. There are two types of electrodes used for invasive brain body interfaces. If signals needed to be obtained with the least noise and from one or few neurons, neurotrophic electrodes were used [11], other choice was Utah Intracranial Electrode Array (UIEA), which contains 100 penetrating silicon electrodes, placed on the surface of cortex with needles penetrating into the brain, which can be used for recording and simulating neurons [12]. Neuron discrimination (choice of single or a group of neurons) does not play any part processing of signals in brain body interfaces [13].

A non-invasive assistive technology device named Cyberlink ${ }^{\mathrm{TM}}$ was used for this research. Only limited amount of research has been done using Cyberlink ${ }^{\mathrm{TM}}$ as the brain body interface. The Cyberlink ${ }^{\mathrm{TM}}$ used in our research, is a brain-body actuated control technology that combines eye-movement (Electrooculargraphy or EOG), facial muscle (Electromyography or EMG) and brain wave (Electroencephalalography or EEG) bio-potentials detected at the user's forehead. Having considered various assistive devices for our research, we chose the Cyberlink as the best device for brain-injured quadriplegic nonverbal participants, since it was non-invasive without any medical intervention and easy to set-up. Previous work done in this area by the researcher has been well documented indicating the challenges involved in this research [14-19].

\section{Chosen Methodology}

Having considered the research methodologies on offer the appropriate one for this investigation was chosen, where the final artefact was evaluated by a small number of severely brain-injured participants [20]. A medical practitioner chose suitable braininjured participants for the research analysing their responses and medication. Comatose and medication that restricted response were used as the criteria for exclusion from this research. 
The approach chosen is shown in diagrammatic form in figure 2. The diagram shows the three phases of the research and the iterative processes that were used to develop the paradigms. The iterative processes that were employed in the design and development of the novel interaction paradigms are shown on the left of the diagram and the other issues that influenced the processes are shown on the right side of the diagram. Iteration driven by phenomenological formative and summative evaluations [21], gives the opportunity for building artefacts that can evolve into refined, tried and tested end products when developing artefacts [22]. The final feedback from each phase is shown in the text boxes in figure 2. One method of conducting scientific research in a new area of study with a new tool is to use the tool with a group of participants and to collect data from the performance of tasks with the tool. The data then display trends that allow other questions to be formed. These questions can be used to form a hypothesis that may be evaluated in further experiments. This method is known as Naturalistic Inquiry [23]. Williams states "naturalistic inquiry is disciplined inquiry conducted in natural settings (in the field of interest, not in laboratories), using natural methods (observation, interviewing, thinking, reading, writing)". Naturalistic inquires were used in this research for investigating topics of interest. Formative research methods and empirical summative methods were used to evaluate the paradigms being investigated in this research [24]. Developed prototypes were tested using able users as test subjects before being evaluated with disabled users. Iteration allowed better feedback for faster interface development. Many versions of the interface program were developed to get the final artefact. Formative method or formative evaluation can be conducted during the planning and delivery of research. This method is based on scientific knowledge based on application of logic and reasoning. It produces information that is used to improve a program while it is in progress.

First phase of the research aimed to replicate Doherty's work with his tunnel interface [19]. Once replicated, a small change, adding discrete acceleration to cursor movement, was made to the interface that greatly improved performance overall. However, this change was not enough to make the most of the wide variations in capability in the user population. This meant that the users could not be grouped according to their disability classification but every user had to have an individually personalised interface [19]. The second phase incorporated discrete acceleration into a more flexible and personalised interface (Fig. 2). It also introduced a control system, which controlled the movements of the cursor by dividing the computer screen into configurable tiles and delaying the cursor at each tile. This new paradigm also brought the cursor back to a starting point after an elapsed period of time, avoiding any user frustration. Able-bodied participants evaluated this paradigm to obtain optimum settings that can be used in phase three thus avoiding any unnecessary training. Re-configuration facility was available for users by running the target test again and replacing the previous personalised interface. The third phase evaluated the novel interface paradigm developed in phase two incorporating the optimum settings. This novel interface paradigm was evaluated with the disabled participants. This proved to be usable by a larger percentage of brain-injured population than in previous Doherty's studies, and over a wider range of functionality. 


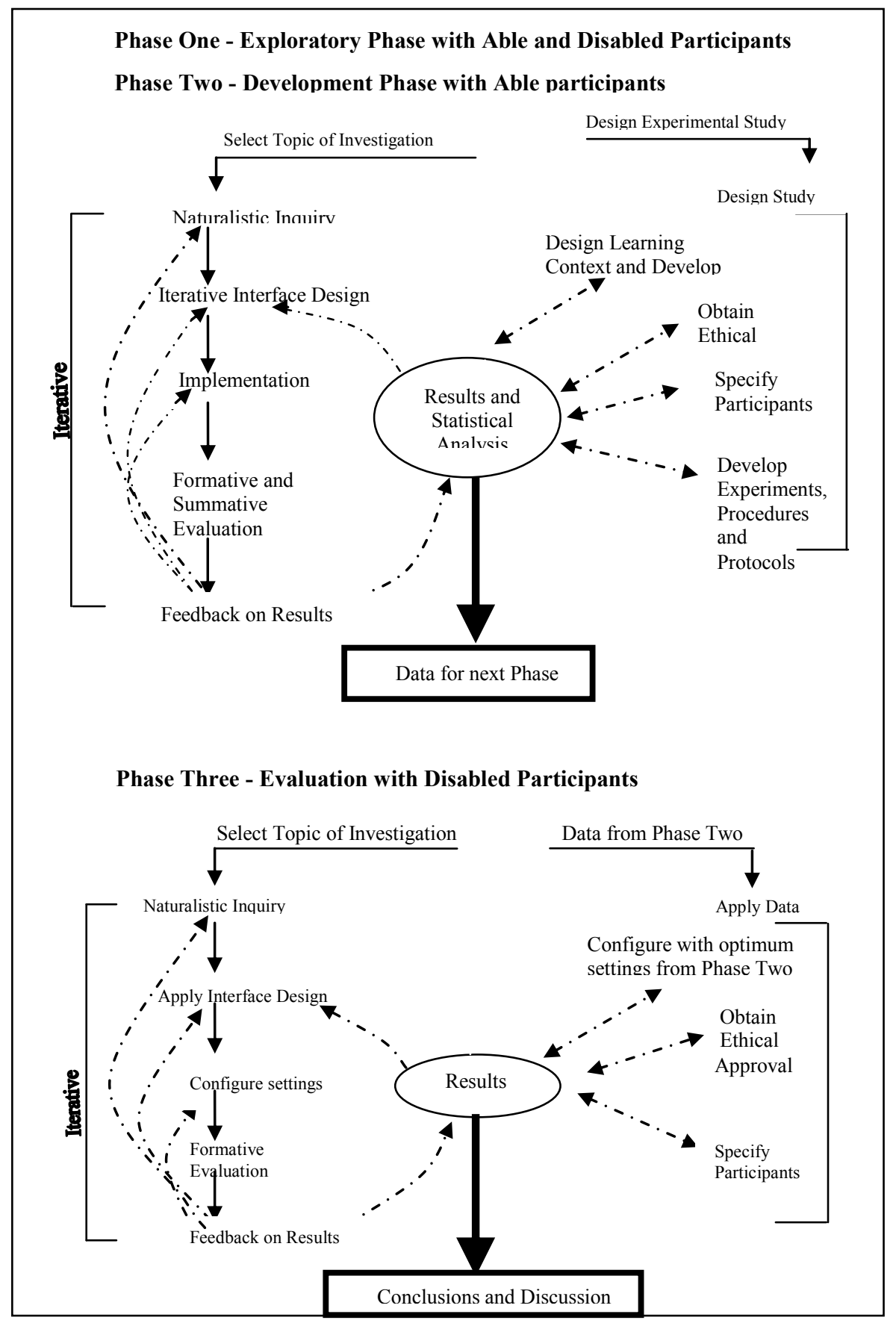

Fig. 2. Chosen Research Methodology 


\section{Developed Interface}

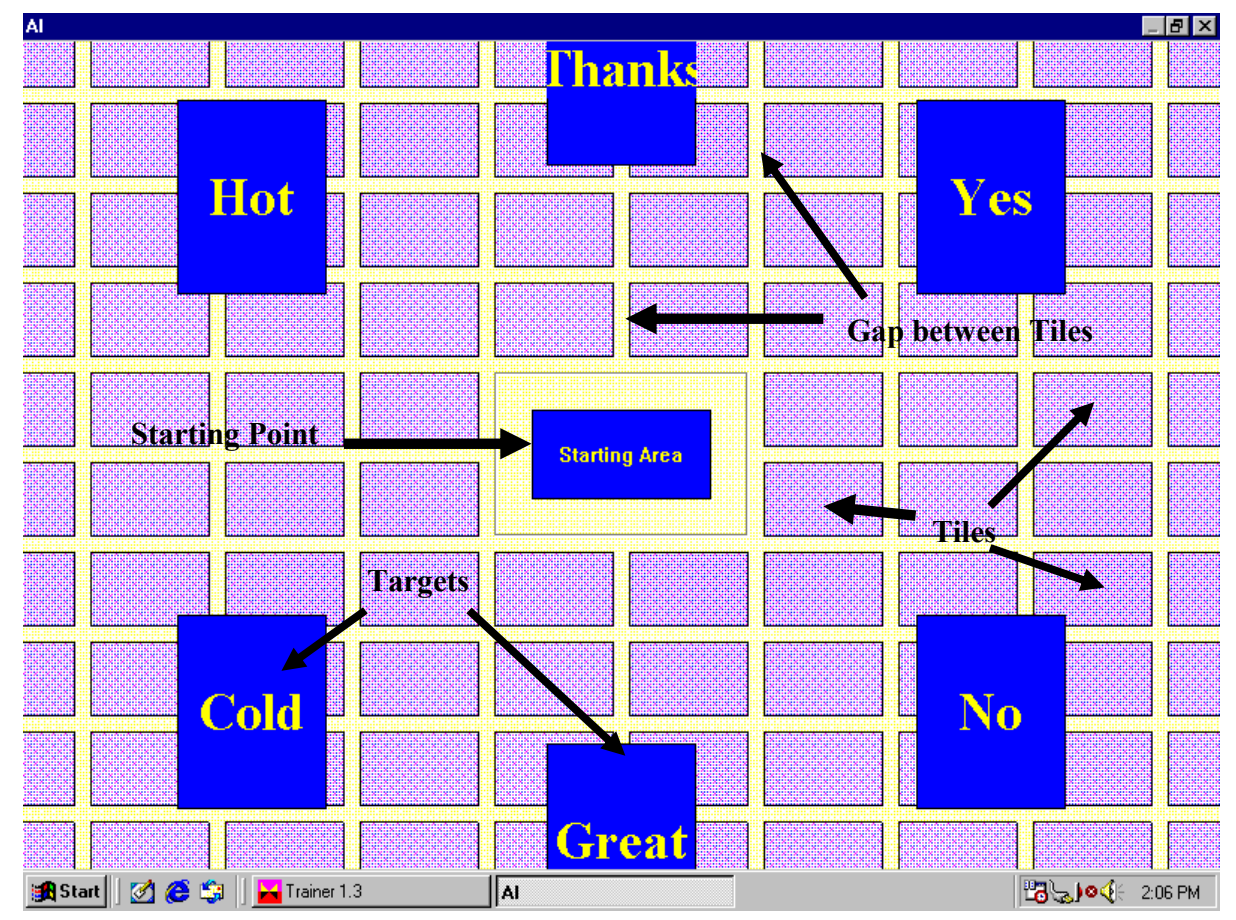

Fig. 3. Interface Used for this research

Prototypes were used for this study from previous research. Fig. 2 shows an example of this interface. The interface was tested with the able participants then disabled participants, using the individual abilities and bio-potentials that could be used. If a disabled user moves a cursor in any direction consistently we were able to create an individual interface and communicate effectively. The initial tests with the disabled participants were to find out how much EEG, EOG or EMG that can be harnessed. The severity of the brain injury of the participants gave only EEG signal for communicating.

In order to support discrete acceleration, the computer screen is divided into tiles, which support discrete jumps from one tile to the next predicted tile on the user's route. However, the lack of regularity in user's cursor paths in study one ruled out a wholly adaptive algorithm, with the following algorithm being implemented instead: The configuration took care of all timings, there were individual times allocated for every task, which mean the interface automatically recovered to the original position (i.e. starting point in the middle) this taking care of error recovery.

The above is still however a universal design that only takes account of user differences at run-time. Irregularities in user input rule out jumping directly to the nearest predicted target. Instead, a step by step approach is taken that leaves the user in control at each point. A wholly automated approach would introduce high error 
recovery costs given the limited capabilities of the traumatic brain-injured. Thus, the interface has further features that allow the cursor's path to be controlled by settings for a specific user. The personalised settings include time spent on the starting area to relax the user before navigating to a target, time spent on each tile to control the biopotential in such a way controlled navigation can take place, size of tile to suit each user etc.

\section{Experiments and Results}

The approach chosen was iteration driven by phenomenological formative and summative evaluations, which gives the opportunity for building artefacts that can evolve into refined, tried and tested end products when developing artefacts. Formative approaches are based on the worldview belief that reality based on perceptions is different for each person. Formative research has to be systematic and subjective, indicating the experience of individual users. Formative and summative methods compliment each other since they generate different types of data that can be used when developing interfaces. Results obtained in summative methods should be tested using statistical methods, statistical significance, hypothesis validation, null hypothesis etc. Previous research [18] showed how five out of ten were unable to participate due to the visual impairment.

Table 1. Previous results with brain injured participants

\begin{tabular}{|l|l|l|l|}
\hline Participant & $\begin{array}{l}\text { Used text } \\
\text { to audio }\end{array}$ & $\begin{array}{l}\text { Launched } \\
\text { Applications }\end{array}$ & $\begin{array}{l}\text { Switched } \\
\text { Devices }\end{array}$ \\
\hline $1,2,3,6,7$ & \multicolumn{2}{|l|}{ No (due to visual impairment) } \\
\hline 5,10 & Yes & No & No \\
\hline $4,8,9$ & Yes & Yes & Yes \\
\hline
\end{tabular}

This new research conducted at the Low Vision Unit of the National Eye Hospital (Colombo) for participants aged between seven and seventy were able to say 'yes' or 'no' using the brain body interface with seventy five percent consistency. The numbers of participants were eight and seven participants were able to use the brain body interface. Overall a maximum of twenty minutes was spent with each participant, of which the first few minutes were used to relax the participants and relieve or at least reduce muscle tension. Then forehead muscles were used to move the cursor of a computer to indicate 'yes' and 'no', to the questions being asked using the interface shown in Fig. 2. Although certain tensed participants needed guidance and help seven out of eight participants could control the curser to say yes and no by frowning and relaxing (using electromyography). 


\section{Conclusions and Future}

A flexible interface was developed to suit each person, with targets positioned by either using the target test program or manually placing them where participants wish. As a result, it has been possible to extend effective interaction for some users to tasks beyond simple communication. This was achieved with less need for adjusting the Cyberlink $^{\mathrm{TM}}$ settings before use. Brain-body interfaces for rehabilitation are still in their infancy, but we believe that our work could be the basis for their more widespread use in extensively extending the activities of severely impaired individuals. It is possible to see this as the main current viable application of brainbody interfaces, since anyone who can use a more reliable and efficient alternative input device should do so.

Vision impaired participants and comatose participants were the two groups of non-verbal quadriplegic brain-injured people who could not be included in the previous study. But exploratory study showed how the vision impaired could also now be included in using brain body interfaces to communicate in the future.

At present the researchers are working in three areas. Exploratory work is being been done for blind participants navigate computer screen using musical guidance. Research is also being carried out on rehabilitation robotics for the brain injured.

Acknowledgments: Dr. Chris Bloor, Professor Gilbert Cockton, Dr. Ivan Jordanov, Dr. Eamon Doherty and to the following institutes Vimhans Delhi, Mother Theresa's Mission of Charity Delhi, Holy Cross Hospital Surrey, Castel Froma, Leamington spa and Low Vision Unit of the National Eye Hospital Colombo.

\section{References}

1. World Health Organization, (2005), Disability, Including Prevention, Management and rehabilitation, Report by the Secretariat document A58/17, April 2005, World Health Organization Publications.

2. Council of Europe, (2002), Towards full social inclusion of persons with disabilities, Report Social, Health and Family Affairs Committee, Doc. 9632, December 2002, Communication Unit of the Assembly

3. Sears, A., Young, M., (2003), Physical Disabilities and Computing Technologies: An Analysis of Impairments, The Human-Computer Interaction Handbook, Jacko, J.A., Sears, A., (Editors), Lawrence Erlbaum Associates, 482 - 503.

4. Roy, E. A., (2004), The anatomy of a head injury, http://www.ahs.uwaterloo.ca/ cahr/headfall.html, accessed $1^{\text {st }}$ May 2005.

5. Kozelka, J., Pedley, T., (1990), Beta and MU Rhythms, Journal of Clinical Neurophysiology, New York1990, 7(2), Raven Press Ltd., 191-207

6. Vaughan et al. (2003), Brain-Computer Interface Technology: A Review of the Second International Meeting, IEEE Transactions on Neural Systems and Rehabilitation Engineering, 11(2), June 2003, 94 - 109.

7. Wolpaw, J., Birbaumer, N., Heetderks, W. J., McFarland, D. J., Peckham, P. H., Schalk, G., Donchin, E., Quatrano, L. A., Robinson, C. J., (2000), Wolpaw, J., Vaughan, T., (Editors), Brain-Computer Interface technology: A review of the First International Meeting, IEEE Transactions on Rehabilitation Engineering, 8(2), June 2000, 164 - 173. 
8. Pregenzer, M., Pfurtscheller, G., Flotzinger, D., (1994), Selection of electrode positions for an EEG-based Brain Computer Interface (BCI), Biomedizinische Technik, Vol.39, 1994, $264-269$.

9. Berg, C., Junker, A., Rothman, A., Leininger, R. (1998), The Cyberlink Interface: Development of A Hands-Free Continuous/Discrete Multi-Channel Computer Input Device, Small Business Innovation Research Program (SBIR) Phase II Final Report, Brain Actuated Technologies, Ohio, USA.

10.Jasper, H., (1958), The Ten-Twenty Electrode System of the International Federation in Electroencephalography and Clinical Neurophysiology, Electroencephalographic Clinical Neurophysiology, Vol. 10, 1958, 371-5.

11.Siuru, B., (1999), A brain /Computer Interface, Electronics Now, March 1999, 70(3), $55-56$.

12.Spiers, A., Warwick, K., Mark Gasson, M., (2005), Assessment of Invasive Neural Implant Technology, July 2005, Lawrence Erlbaum Associates, HCI International 2005, Las Vegas, CD-ROM.

13.Sanchez, J. C., Principe, J. C., Carne, P.R., (2005), Is Neuron Discrimination Preprocessing Necessary for Linear and Nonlinear Brain Machine Interface Models? Lawrence Erlbaum Associates, HCI International 2005, Las Vegas, CD-ROM.

14.Gnanayutham, P., (2004), Assistive Technologies for Traumatic Brain injury, September 2004, ACM SIGACCESS Newsletter, issue no. 80, pages 18 - 21.

15.Gnanayutham, P., (2006), The State of Brain Body Interface Devices, UsabilityNews, http://www.usabilitynews.com/, October 2006.

16.Gnanayutham, P., Bloor, C., Cockton, G., (2001), Robotics for the brain injured: An interface for the brain injured person to operate a robotic arm, Edited by Antoniou. G., Deremer. D., October 2001, ICCIT'2001, New York, 93 - 98

17.Gnanayutham, P., Bloor, C., Cockton, G., (2003), AI to enhance a brain computer interface, Edited by Stephanidis, C., 1397 - 1401, June 2003, Lawrence Erlbaum Associates, HCI International 2003, Crete.

18.Gnanayutham, P., Bloor, C., Cockton, G., (2005), Discrete Acceleration and Personalised

18.Tiling as Brain-Body Interface Paradigms for Neurorehabilitation, CHI 2005, April 2005, ACM Press, Portland, Oregon, $261-270$.

19. Gnanayutham, P., (2005), Personalised Tiling Paradigm for Motor Impaired Users, HCI International 2005, July 2005, Lawrence Erlbaum Associates, Las Vegas. CD-ROM

20.Preece, J., Rogers, Y., Sharp, H., (2002) Interaction Design, Wiley, USA

21.Munhall, P. L., (1989) Philosophical ponderings on qualitative research methods in nursing, Nursing Science Quarterly, 2(1), 20 - 28

22.Abowd, G., Bowen, J., Dix, A., Harrison, M., Took, R., (1989), User Interface Languages: a survey of existing methods, Oxford University Computing Laboratory, Programming Research Group, October 1989, UK.

23.Williams, D.D., (1986). When is Naturalistic Evaluation Appropriate? Williams, D, D., (Editor) Naturalistic Evaluation. New Directions for Program Evaluation, no. 30. San Francisco: Jossey-Bass.

24.Nogueira, J. L., Garcia, A. C. B., (2003), Understanding the Tradeoffs of Interface Evaluation Methods, Edited by Jacko, J. and Stephanidis, C., 676 - 680, June 2003, HCI International 2003, Crete. 